\title{
Experimental Study on Self-propelled Motion of Leidenfrost Droplets
}

\author{
Cuiping $\mathrm{Xu}^{1}$, Jiayu Zhang ${ }^{2}$, Fan Zhang ${ }^{2}$, Chengbin Zhang ${ }^{2}$ \\ ${ }^{1}$ Chien-Shiung Wu College, Southeast University, Nanjing 210096, China \\ 213161011@seu.edu.cn \\ ${ }^{2}$ Key Laboratory of Energy Thermal Conversion and Control of Ministry of Education, School of Energy and \\ Environment, Southeast University, Nanjing 210096, China \\ 213162206@seu.edu.cn; 213163059@ seu.edu.cn; cbzhang@ seu.edu.cn
}

\section{Extended Abstract}

Microfluidic technology has great potential application in biomedical engineering, electronic cooling, etc. The manipulation ability to droplets is one of the core issues in the real application. Due to the Leidenfrost phenomenon, a single liquid droplet with a vapor layer can forms on the solid wall with high temperature. The presence of vapor layer obstructs the heat exchange between the droplet and the wall surface, and hence slows down the evaporation rate of the droplets [1]. Arising from the Leidenfrost phenomenon, the droplets can self-propel on a solid wall along a well-defined direction. Nowadays the Leidenfrost mechanism and the droplet self-motion are understood, however, the interface evolution, formation mechanism, motion characteristics of the Leidenfrost droplets self-propelled motion under the serrated surface with different temperature conditions have not been revealed completely. In this context, we designed the solid surfaces for droplet self-motion, and the experimental platform for visualizing the evolution of the droplet self-propelled motion phenomenon is set up, then the droplet boiling phenomenon and the Leidenfrost self-propelled dynamics on the droplet selfdriven surface is observed with the help of the high-speed micro-imaging system. In the experiment, two kinds of liquid, i.e. ethanol and water, are adopted as the working media. The experimental data were extracted to analyze the interactions between droplet film boiling super-heat, heat flux density, critical temperature, self-motion characteristics and surface properties, droplet morphology. The results indicated that the self-propelled velocity and stability of different droplets at the Leidenfrost temperature point are different; the motion velocity of ethanol is slower than that of water, but it is more stable than water; the droplets volume also affects the motion velocity of droplet. When the diameter of the droplet is 2 or 3 times larger than that of a single sawtooth, the velocity of the droplet is faster. Besides, the droplets have the ability to climb against gravity. By this visualization experiment, we can analyze the droplet self-propelled motion more systematically and intuitively, provide more convenience for the study of droplet manipulation.

\section{Acknowledgement}

This work was supported by National Natural Science Foundation of China (Grant No. 51776037 and U1737104).

\section{References}

[1] A. L. Biance, C. Clanet and D. Quéré, "Leidenfrost drops," Phys. Fluids, vol. 15, no. 6, pp. 1632-1637, 2003. 